\title{
HISTORIA
}

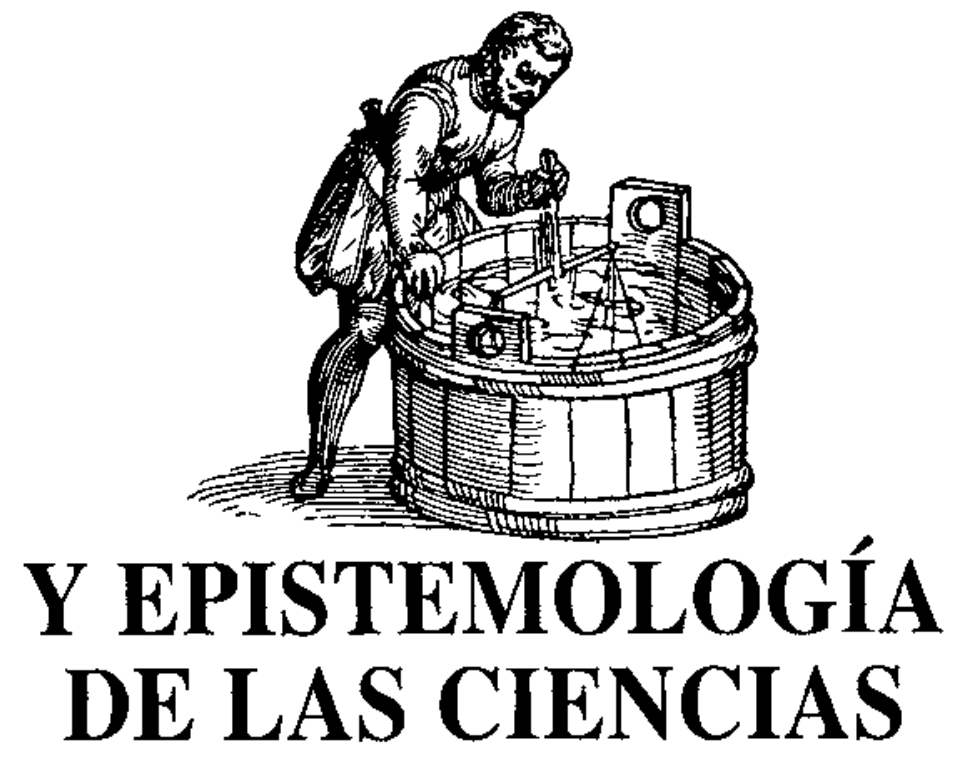

\section{DEFICIENCIAS EPISTEMOLÓGICAS EN LA ENSEÑANZA HABITUAL DE LOS CONCEPTOS DE CAMPO Y POTENCIAL ELÉCTRICO}

\author{
FURIÓ, C. ${ }^{1}$ y GUISASOLA J.2 ${ }^{2}$ \\ ${ }_{1}$ Departamento de Didáctica de las Ciencias Experimentales. Universidad de Valencia. \\ ${ }^{2}$ Departamento de Física Aplicada I. Universidad del País Vasco.
}

\section{SUMMARY}

This article intends to analyse up to what extent the scarce learning produced when introducing the basic concepts on electrostatics is due to deficiencies and inadequacies in its routine teaching. So, on the one hand, we analyse what have been the principal epistemological leaps forward in the making of electricity as a science. On the other hand, we analyse Physics books and the teachers' opinions.

\section{INTRODUCCIÓN}

EI problema de las dificultades que tienen los estudiantes en el aprendizaje de los conceptos de campo y potencial eléctrico es bien conocido por los profesores de física de bachillerato y $I x$. ciclo de las facultades de 
ciencias. Asimismo, dentro de las investigaciones sobre concepciones alternativas, las dificultades de aprendizaje de las principales nociones de electricidad, tales como la carga, el potencial eléctrico o la corriente eléctrica, están siendo investigadas extensamente en el nivel de la enseñanza secundaria. Estas investigaciones se han centrado más en el dominio de la electrocinética (Duit, 1993) $y$, en particular, en el estudio de los circuitos eléctricos de corriente continua sin que, normalmente, se establezcan relaciones con la electrostática y, especialmente, con el concepto de campo eléctrico, que es funcamental para entender el movimiento de cargas (Eylon y Ganiel, 1990). Los trabajos que tratan sobre el aprendizaje del concepto de campo (Nardi y Carvhalo, 1990; Solbes y Martin, 1991; Viennot y Rainson, 1992; Guisasola y Furió, 1994; Galiti, 1995) muestran que Ios estudiantes presentan serias dificultades en su aplicación a situaciones de electrostática, donde deben utilizar nociones básicas de la teoría como el principio de superposición, tener en cuenta el papel que juega el medio, el concepto campo-interacción, etc.

Este trabajo de investigación se sitúa dentro de una orientación constructivista, que se caracteriza básicamente por considerar el aprendizaje como construcción activa de nuevo conocimiento por parte del aprendiz, mediante la interacción de sus estructuras mentales con la información que recibe del exterior y en la cual construcción las iđeas previas juegan un papel relevante (Driver, 1989; Resnick, 1983). Esta forma de entender el aprendizaje ha llevado a la aparición de propuestas de intervención didáctica que, en un principio, tuvieron como denominador común el uso de estrategias basadas en el cambio conceptual (Posner et al., 1982). Sin embargo, las nuevas aportaciones de la historia y filosofía de la ciencia nos indican que, cuando se producen cambios o revoluciones científicas, no sólo hay cambios conceptuales en la vieja teoría sino que también se producen cambios en las formas de ver el mundo (cambios ontológicos), en las formas de razonar (cambios epistemológicos), en los métodos (cambios metodológicos) y en los propios valores y propósitos de la nueva teoría (cambios axiológicos) (Dusch1 y Gitomer, 1991; Chi, 1991; Mortimer, 1995). En el caso de asimilar el proceso de construcción de conocimientos en la ciencia con el de aprendizaje cientifico, se puede extrapolar que este último proceso no puede reducirse a un simple cambio conceptual sino que también tiene sus exigencias ontologicas, epistemológicas y metodológicas. Esto ha originado la aparición de nuevos modelos de enseñanzaaprendizaje que asocian explícitamente el cambio conceptual con cambios epistemológicos y metodológicos en la construcción de conocimientos, tales como la «cultura del portafolion (Duschl y Gitomer, 1991) o el «aprendizaje por investigación» (Gil, 1993; Porlan, 1993; Furió, 1994).

En este contexto constructivista, el problema didáctico que nos planteamos en este trabajo es el siguiente:

¿En qué medida, el poco aprendizaje producido al introducir en el bachillerato los conceptos básicos de electrostática (campo y potencial eléctricos) puede ser debi- do a carencias y deficiencias epistemológicas de las estrategias de transmisión verbal empleadas en la enseñanza habitual?

Una de las aportaciones de la historia y epistemología de la ciencia es que nos permite establecer las características fundamentales de la naturaleza de la ciencia y el trabajo científico (Matthews, 1990; Cleminson, 1990; Giannetto et al., 1992). Estas características nos permitirán detectar las visiones distorsionadas de la ciencia y del trabajo científico que se pueden dar en la enseñanza habitual. Así pues, una posible respuesta al interrogante planteado pasará, en primer lugar, por un análisis cuidadoso de aquellas teorías que por su importancia representaron verdaderos saltos cualitativos en la construcción de la electricidad, centrándose, en particular, en aquellas dificultades y problemas cuya superación determinó el avance científico (Furió y Guisasola, 1993). Por tanto, se comenzará por examinar, desde un punto de vista conceptual, los saltos cualitativos más importantes en el desarrollo de la teoría eléctrica en los siglos XVIII y xIx (Holton y Roller, 1962; Heilbron, 1979; Nerssesian, 1995; Whittaker, 1987).

\section{PRINCIPALES APORTACIONES EPISTE- MOLOGICAS A LA CONSTRUCCIÓN DE IA ELECTRICIDAD EN LOS SIGLOS XVIII Y XIX}

\section{La evolución de la teoría eléctrica a lo largo del siglo XVIII}

A mediados del siglo xvin la palabra electricidad empezó a significar que «una sustancia of fuido que se suponía que estaba dentro de los cuerpos cuando se electrizaban». Este significado venía apoyado, por un lado, por los experimentos -era muy difícil evitar la idea de que algún tipo de fluido se desplazaba cuando, al frotar un tubo de vidrio conectado con una línea de varios metros, quedaba electrizado el extremo de la línea y, por otro lado, por el lenguaje utilizado por investigadores anteriores como Gray y Du Fay. Así mismo, el mecanicismo newtoniano estaba en pleno apogeo y su influencia «atomista» se extendía a muchas ramas de la ciencia como, por ejemplo, al atribuir carácter sustancial (fluido) al calórico o al flogisto.

La aportación científica de B. Iranklin (1706-1790) constituyó una de las mayores contribuciones del siglo xvin a la explicación de los fenómenos eléctricos (Heilbron, 1979). Franklin mantuvo una correspondencia continuada, en la que iba comunicando sus descubrimientos, con su amigo Peter Collins. Estas cartas fueron publicadas en Londres bajo el título de «Experiment and Observations on Electricity». En su carta II (1747) proponía como hipotesis su «modelo de fluidon para los fenómenos eléctricos. Éste consistía en considerar que todos los cuerpos contienen una cantidad de fluido (electric fire) en su estado neutro. Se definía este fluido 
O «Sustancia eléctrica» como un conjunto de partículas que se repclían mutuamente y que podían penetrar en Ia matcria ordinaria (Franklin, 1988). El modelo así enunciado permitía explicar los fenómenos eléctricos descubiertos en la época, como la electrización por frotamiento, las atracciones y repulsiones eléctricas y la conducción eléctrica. Por ejemplo, Ia electrización por frotamiento se explica suponiendo que el cuerpo frotado adquiere una cantidad extra de fluido o pierde parte de su cantidad "normal» de fluido, de forma que el cucrpo queda cargado «positiva o negativamente». El hecho dc que el modelo afirme que todas las electrizaciones se deben a un movimiento de materia eléctrica -lo que gana un cuerpo lo pierde el otro- implica aceptar no sólo la transferencia sino también la noción de conservación de la carga.

Posteriormente W. Watson (1715-1787) introduce como causa del movimiento del fluido eléctrico su diferencia de densidades. Esta idea supuso un salto cualitativo, ya que implica atribuir al fluido eléctrico una cierta compresibilidad (modelo gaseoso). Esta presión del fluido eléctrico fue considerada como un primer concepto cualitativo que puede preceder a lo que hoy entendemos por diferencia de potencial y lo encontraremos más tarde en la explicación đada por el propio Ohm a su famosa ley (Benseghir, 1987).

Otro aspecto a destacar en la teoría de Franklin es la confusión de los conceptos de electricidad, o materia eléctrica, y la atmósfera cléctrica que rodea a los cuerpos cargados, es decir, lo que hoy conocemos como su campo de fuerza. Esta confusión era corriente a mediados del siglo xvIII; se encuentra tambien en los trabajos de sus contemporáneos W. Watson (1715-1787) y el abate Nollet (1700-1770) (Rodríguez, 1983). Sin embargo, los trabajos de Coulomb y la impregnación definitiva de las ideas newtonianas en la electricidad harán abandonar por completo la idea de atmósfera elécirica. Volverá a aparecer con Faraday, pero muy modificada, muchísimo más precisá y más directamente relacionada con la experiencia.

Hacia el último tercio del siglo xvili se opinaba que para un rápido avance del estudio de la electricidad era neccsario establecer bases cuantitativas y definir magnitudes de forma operativa. A pesar de que Franklin había introducido el concepto de carga eléctrica o «cantidad de sustancia eléctricas, ni él ni sus sucesores fucron capaces de medirla. En este sentido, de nuevo se pone de manifiesto la importancia de la metodología científica, ya que, como es sabido, para diseñar experimentos de medida de un concepto es necesario una hipótesis que los guíc y, este era precisamente el problema que tenían los sucesorci de Franklin. En tales circustancias tuvieron que tomar como referencia alguna otra parte de la cicncia más desarrollada, la cual, además de poseer conceptos fundamentales definidos de forma opcrativa, tratase de fenómenos de características similares a los de la electricidad. De esta forma investigadores como Cavendish, Priestley y Coulomb buscaron una teoría análoga a Ia gravitación, bajo la clara influencia del mecanicismo newtoniano (Taton, 1988).
Según indica Whittaker (1987, pp. 53), Priestley, en su libro The history and present state of electricity, with original experiments, publicado en 1767, comenta el hecho de que las bolas de corcho, al ser introducidas en un recipiente de metal cargado eléctricamente, no eran afectadas en absoluto por la electricidad; y emite la siguiente hipótesis:

"No puede inferirse de este experimento que lat atracción de la electricidad está sometida a las mismas leycs de la gravitación y que sigue, por consiguiente, los cuadrados de las distancias, puesto que puede demostrarse que, si la Tierra fuera una concha hueca, un cuerpo que estuviera en su interior no seria atraído hacia un lado más que hacia cl otro?» (Traducción de los atutores).

En 1785 aparece la primera memoria de Coulomb sobre electricidad (Coulomb, 1884), a la que seguirán otras hasta 1788. En ellas Coulomb relaciona por primera vez la «masa eléctrica», es decir, la carga eléctrica, en valores absolutos con magnitudes mecánicas fundamentales de forma que puede ser sometida a cálculos.

El nuevo modelo que surge es un modelo mecanicista que define la carga de forma operativa a partir de sus efectos dinámicos (según la ley de Coulomb) y no se define sobre el modelo de fluido único de Franklin. El modelo, de forma coherente con la física de Newton, introduce «la acción a distancia», que actúa de forma instantánea entre los cuerpos cargados, siendo las fuerzas de tipo central calculables con arreglo a la ley mencionada. De hecho, los científicos imaginaban ahora el fluido eléctrico constituido por partículas eléctricas que permanecían en el cuerpo durante las atracciones y repulsiones, ejerciéndose fuerzas entre sí a distancia sin intermedio de ningún soporte material.

Así pues, la teoría de Coulomb extendía la mecánica newtoniana a la electricidad. De esta forma, se acepta el modelo que subyace en la mecánica newtoniana, que en cierta medida es comparabie a la teoría de Demócrito: el mundo está constituido por "corpúsculos" sólidos y extensos, y por espacio vacío. Sin embargo, en la teoría de Newton se introduce una tercera entidad, Ia fuerza. Cada «corpúsculo» posee Ia propiedad de «actuar a distancia» y ejercer fuerzas directas e instantáneas sobre otros cuerpos del universo. Como dice W. Berkson (1985), no está claro que la concepción descrita fuera «realmente» la de Newton, pero sí creemos que el modelo expuesto es básicamente similar al que se atribuye a Newton justificada o injustificadamente.

\section{La ciencia de la electricidad a comienzos del siglo XIX}

A comienzos del siglo xix los físicos habían adquirido la costumbre de utilizar también en el dominio de la electricidad et modelo de «acción a distancia», ya que trabajan con la teoría de Coulomb que extendió a finales del siglo anterior la mecánica newtoniana a este dominio. Sin embargo, Ia física del siglo XIX se caracteriza por la introducción de un nuevo concepto, el de energía, que intenta unificar las diferentes fuerzas existentes en la 
naturaleza (mecánica, eléctrica, química, magnética...) (Harman, 1990). Este impulso dado a la creación de una física unificada fue influenciado por diversas corrientes filosóficas, científicas e incluso literarias (Thuillier, 1989) que divergían del mecanicismo newioniano tanto respecto de la visión del mundo como de la forma en que los humanos conocemos dicho mundo (Mason, 1985).

Nuevos hechos experimentales se explicaban mediante mecanismos interactivos diferentes a la «acción a distancia» como, por ejemplo, la teoría ondulatoria de Fresnel, que suponía que la luz se propagaba mediante las vibraciones de un éter mecánico o, por ejemplo, la conversión de la «fuerza eléctrica» en «fuerza química», que se empleaba para justificar por qué la electricidad voltaica podría descomponer sustancias químicas. Finalmente cl experimento de Oersted no dejaba dudas sobre las relaciones entre electricidad y magnetismo, viniendo a corroborar esta «transformación de fuerzas», que no cabían en el esquema newioniano.

\section{La transición de un modelo de acción a distancia a un modelo de campo}

En tos trabajos de Ampère se intentaba explicar las relaciones entre fuerzas eléctricas y magnéticas con hipótesis basadas en el punto de vista newtoniano, mediante una ingeniosa teoría matemática de la atracción entre corrientes que daba cuenta de los resultados experimentales hasta entonces conocidos. Sin embargo, fue Faraday el primero en sugerir que la acción a distancia resulaba inadecuada para dar cuenta de estas interacciones.

En efecto, Faraday dudaba de que la electricidad estuviese constituida por una sustancia ffuida o dos flujos. Crcía más bien que se trataba de un estado especial de las fuerzas de un cuerpo cargado. Faraday «veía» en las explicaciones que Oersted daba a su experimento «un campo de fuerza». Así lo comenta en I82l en la publicación Historical Sketch of Electromagnetism:

«El conflicto eléctrico no se encuentra confinado en el conductor, sino que está muy extendido por el espacio circundante, ya que en caso contrario no actuaría a distancia sobre la aguja de una brújula.» (Traducción de los autores).

Posteriormente, a partir del descubrimiento de la inducción magnética, Faraday fundamenta empíricamente su concepción de la materia y construye su teoría de las líneas de campo magnético. Su concepción del mundo puede resumirse diciendo que la fuerza es una propicdad universal que se extiende a lo largo del espacio; a cada punto del campo de fuerza se le asocia una intensidad y una dirección. De forma que, según sea la intensidad y la dirección de la fuerza, el punto de fuerza hará que los puntos vecinos se muevan. Por tanto, todos los puntos del sistema interactúan con sus vecinos, dando lugar a todas las posibles distribuciones de fuerza y vibraciones de ésta (Berkson, 1985).
El campo de fuerza venía definido a través del concepto de líneas del campo, que en su trabajo Experimental Researches on Electricity (Faraday, 1839) enuncia de la siguiente forma:

«Cuando un hilo se mucve directa u oblicuamente a través de las líneas de fuerza [...] suma la cantidad de fuerzas representada por las líneas que ha cortado [...] la cantidad de electricidad que pasa a la corriente es proporcional al número de líneas cortadas.”

Más adelante precisa su idea de líneas de fueræa:

«La cantidad relativa de fuerza en un punto [...] en un espacio dado, está indicada por la concentración o separación de la líneas.”

El estudio de fenómenos de inducción eléctrica confirmó la hipótesis de Faraday de que las fuerzas eléctricas se propagaban a través de un medio, y le Ilevaron a la conclusión de que era el medio el que propagaba el fenómeno de la inducción eléctrica entre conductores. Las propias partículas del medio actuaban como conductor al quedar polarizadas. Esta acción tenía lugar entre partículas contiguas y a lo largo de líneas curvas, $k$ que para Faraday era una prueba concluyente de que la inducción no era fruto de una acción a distancia sino de una acción continua a través del medio.

\section{EI cambio ontológico que supuso la utilización de la idea de campo}

El modelo que presenta Faraday en sus «Investigaciones experimentales de electricidad», que fueron publicadas por él mismo en 1839, se basa en dos ideas claves: que la acción de un cuerpo cargado sobre otro separado a una cierta distancia requiere un determinado ticmpo y que la acción se transmite por medio de la perturbación de cada parte del campo sobre la contigua. Fste modelo es coherente con la cosmología cartesiana. Así mismo, la poca relevancia dada por la mecánica newtoniana al medio material existente entre las cargas es coherente con su posición respecto a cómo es el universo. Las características principales de ambas cosmologías han sido resumidas en el cuadro de la página siguiente.

Por otra parte, el modelo de campo supone una nueva forma de ver la interacción eléctrica (cambio ontológico) ya que se debe imaginar ésta sin haber cargas' testigo que la evidencien, e introduciendo el concepto de energía potencial en el contexto de la teoría de campos. Una medida de la dificultad de este modelo vienabien expresada a través de las propias palabras de Maxwell (1865) al referirse al carácter «posicional» del vector intensidad de campo cléctrico en Dinamical theory of the electromagnetic field:

«Al hablar de la intensidad del campo eléctrico resultante en un punto, no suponemos necesariamente que allí sea ejerciđa realmente una fuerza, sino sólo que, si se coloca allí un cuerpo electrizado, actuará sobre él una 


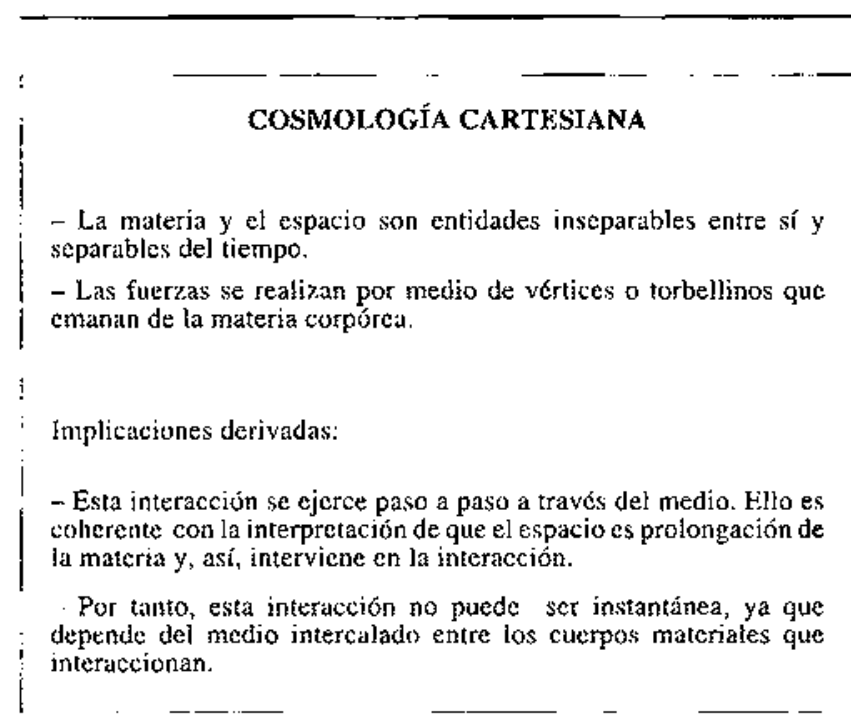

fuerza [...] proporcional a la carga del cuerpo.» (Traducción de los autores).

Es preciso resaltar que la teoría de campo iniciada por Faraday también supone una nueva concepción de la interacción eléctrica, cuya representación no se limita localmente a las partículas materiales cargadas sino que se extiende por el espacio circundante. Profundizando más en cl cambio ontológico que se produce de la visión coulombiana a la de campo eléctrico, podemos decir que en la primera cl concepto de interacción eléctrica está ligado al concepto de carga localizada en una zona del espacio o en un cuerpo cargado. Es decir, en esta visión no podemos concebir la existencia de la interacción eléctrica sin tener localizadas en el espacio las cargas electricas que interaccionan. La nueva visión del campo eléctrico nos obliga a pensar de forma diferente, el concepto de interacción eléctrica deja de estar asociado a dos cargas eléctricas para extenderse a lo largo del espacio de influencia de una de aquéllas. Es decir, si prescindimos de una de estas cargas (por ejemplo, la carga testigo), la intcracción eléctrica puede ser sin que necesariamente se asocic al estar de una carga eléctrica. A partir de esta nueva concepción de la interacción eléctrica es fácil cstablecer la relación entre los conceptos de carga, campo eléctrico y energía potencial eléctrica.

\section{La influencia del modelo de acción a distancia en el desarrollo de la teoría eléctrica a lo largo del siglo xix}

Hacia el cuarto decenio del siglo quedó cerrada, al menos durante algún tiempo, la era de los grandes descubrimientos experimentales en electricidad que se habían sucedido con gran celeridad. Las vías de investigación se volvieron más teóricas debido a la necesidad de justificar los múltiples hechos experimentales. A pesar de la vía de investigación abierta por Faraday que explicaba estos fenómenos basándose en una «propagación paso a paso" de la acción electromagnética, estas

ideas fueron consideradas al principio confusas por muchos científicos que estaban más habituados a la forma de trabajar en la mećánica newtoniana; e inciuso más tarde, cuando Maxwell las precisó en lenguaje matemático, siguieron siendo mal comprendidas durante mucho tiempo (Whittaker, 1987).

Muchos científicos continuaban interpretando los fenómenos electromagnéticos en base al modelo de «acción a distancian y los cálculos se llevaban a cabo mediante los métodos clásicos de la mecánica newtoniana. Essta segunda vía de investigación difería explícitamente del modelo de campo. Esto se muestra, por ejemplo, en el siguiente pasaje de la memoria que escribió Ampère (científico que lideró esta vía de investigación) en 1827 , titulada Theòrie mathématique des phénomènes électrodynamiques uniquement déduite de l'expérience. $\mathrm{Am}$ père inicia su memoria apoyando claramente el modelo newtoniano y acusa a Oersted de seguir un modelo de torbellinos cartesianos.

«El sabio ilustre que ha observado el primero los polos de un imán girados por la acción de un hilo conductor ha concluido que la materia clćctrica daba vueltas alrededor de él, precisamente como Descartes hacía dar vueltas a sus remolinos en el sentido de giro de los planetas.” (Traducción de los autores).

Aunque esta línca de investigación terminó en un callejón sin salida, permitió formular leyes importantes que después fueron integradas por Maxwell en la formulación matemática del modelo de campo. Precisamente la convergencia de algunos de los resultados hizo que existiera cierta confusión respecto al modelo físico a utilizar. Esta situación impidió, hasta la publicación por Maxwell de su teoría y la confirmación experimental de Hertz a finales del siglo, que emergieran definitivamente las virtualidades de la idea de campo para analizar las interacciones electromagnéticas.

En síntesis y de acuerdo con la exposición necesariamente resumida que acabamos de hacer, cl modelo 
newtoniano y el maxwelliano para interpretar las interacciones entre cargas pueden considerarse de distinto estatus ontológico y epistemológico pero no contrarios. Es decir, pueden considerarse como dos perfiles conceptuales diferentes (Mortimer, 1995) a la hora de abordar cl enfoque de la resolución de situaciones problemáticas científicas. Inicialmente se presentan en la historia como teorías opuestas con tradiciones de investigación diferentes (por ejemplo, Ampère y Faraday), con tensiones entre ellas; después manifiestan cierta convergencia al llegar a explicar los mismos hechos con distintas bases teóricas; $y$, finalmente, quedan ambas asumidas por la comunidad científica si bien se reconocen las virtualidades de la nueva teoría. O sea, se valora el mayor estatus ontológico y epistemológico del perfil max welliano pero tambiên se reconoce la mayor simplicidad y sencillez del perfil coulombiano. Es más, muchas veces la construc. ción del nuevo perfil conceptual requiere previamente la adquisición del viejo perf́l (por ejemplo, la introducción del campo eléctrico no es posible sin el conocimiento de los prerrequisitos de carga y fuerza eléctricas coulombiana) y el reconocimiento de sus insuficiencias teóricas.

\section{EMISIÓN DE HIPÓTESIS}

Una vez expuesto de forma resumida el principal salto cualitativo en el desarrollo de la electricidad como ciencia, una posible hipotesis de trabajo plausible es considerar que la enseñan7a habitual de la electrostática presentará carencias ontologicas y epistemológicas que dificulten el aprendizaje significativo de los conceptos básicos de la misma.

La hipótesis que acabamos de esbozar como respuesta al problema didáctico planteado, la podemos enunciar de forma más precisa de la siguiente manera:

Hipótesis: La enseñanza habitual de los conceptos básicos de electricidad (desde las primeras nociones de electrostática hasta la teoría elemental de campo eléctrico) presenta visiones distorsionadas en relación con la construcción de la ciencia y del trabajo científico.

La operativización de la hipótesis implica la enumeración y análisis de diversos aspectos implicados en la enseñanza habitual. Este análisis se ha dirigido hacia los dos protagonistas principales del proceso: los libros de texto y el profesorado. Esto nos permite precisar más la hipótesis, de trabajo en base a consecuencias contrastables. Si fuera cierto lo que se afirma en la hipótesis, entonces la organización y secuenciación de contenidos de los libros de texto y de los profesores presentarían graves carencias ontológicas y epistemologicas que se concretan en:

H.1. Uaa presentación acumulativa acritica de los contenidos está caracterizada por una exposición lineal de los conceptos que componen el modelo teórico actual de la electricidad, de forma que no se tienen en cuenta los saltos cualitativos ni los problemas que dieron lugar a nuevos conceptos de mayor poder explicativo. Así pues, existirá una introducción del concepto de campo eléctrico aproblemática $\mathrm{y}$, por tanto, arbitraria.

H.2. La enseñanza adolecerá de falta de conocimiento del cambio ontológico, que se produjo al pasar de una visión coulombiana de las interacciones eléctricas a otra de campo eléctrico. En consecuencia, esta carencia puede llevar al profesorado a considerar que esta última teoría es muy abstracta, sin mucho contenido físico e innecesaria su introducción en clase.

\section{METODOLOGÍA DE IAA INVESTIGACIÓN Y DISENOS EXPERIMENTALES}

De acuerdo con la hipotesis de trabajo que acabamos de enunciar, en la enseñanza de la electrostática que habitualmente se realiza no se tienen en cuenta las principales características de la metodología científica, ni se aprovechan o explotan la historia y epistemología de la ciencia en el sentido de señalar posibles difícultades epistemologicas que puedan presentarse actualmente a los estudiantes, ni tampoco se aprovechan estos conocimientos para fundamentar una mejor selección y secuenciación de los contenidos del tema (Izquierdo, 1992).

Para contrastar la hipótesis se ha realizado un discño múltiple y convergente que ha consistido en la utilización de técnicas semicuantitativas tales como cuestionarios para el análisis de textos o encuestas, así como cualitativas tales como entrevistas a profesores

Al elaborar los cuestionarios se han realizado sondeos previos y se han validado sometiendo al juicio de expertos (otros profesores e investigadores) tanto su contenido como los criterios de valoración de los objetivos. Asimismo, las entrevistas se han registrado en un cutsete $y$, una vez transcritas, se han analizado por los autores de este trabajo y otros dos expertos, de forma que la coincidencia en las interpretaciones ha sido prácticamente total (Jong, 1995).

Para contrastar que la organización y secuenciación de los contenidos de electrostática en los libros de texto presentan las visiones distorsionadas de la ciencia y del trabajo científico que se han cnunciado en $\mathrm{H} .1$ y H.2, se ha elaborado el documento 1 (Anexo).

Respecto al profesorado, se han diseñado dos tipos de prueba con el objeto de averiguar, por una parte, cómo realizan los profesores la secuenciación de contenidos de electrostática y, por otra, analizar si presentan visiones distorsionadas de la ciencia y el trabajo científico. Más en concreto, las pruebas son las siguientes:

a) La primera ha consistido en confeccionar y pasar un cuestionario a 30 profesores en activo de Física y Química de bachillerato ( $3^{\circ}$ de BUP y COU) y a 30 profesores en formación (realizan el CAP) (Anexo, documento 2). 
b) La segunda prueba ha consistido en entrevistar a una muestra de 6 profesores de bachillerato que imparte Física y Química en $3^{\circ}$ de BUP y Física en COU, y otra muestra de 6 profesores aniversitarios que imparten Física General en primer curso (Anexo, documento 3).

Somos conscientes de que la muestra de profesores de universidad es muy limitada y no permite llegar a definir un «perfil» para el profesorado de este nivel. Sin embargo, el carácter voluntario de la entrevista nos lleva a pensar que son profesores motivados hacia la enseñanza, y que las limitaciones epistemológicas y ontológicas encontradas también las tendrán profesores menos activos profesionalmente.

\section{ANÁLISIS E INTERPRETACIÓN DE LOS RE- SULTADOS}

\section{Resultados del análisis de libros de texto}

Comenzaremos con los resultados obtenidos del análisis de tos libros de texto del documento 1 . Se han revisado treinta libros de texto, veinte de ellos corresponden a niveles de bachillerato editados en la década de los ochenta y noventa (diez de $3^{\circ}$ de BUP y diez de COU), y su elección se ha basado en criterios de difusión en el mercado y en sus enfoques variados. Los otros diez libros de texto correponden a libros de física general que, a nuestro juicio, suelen ser utilizados por el profe- sorado para realizar las programaciones. Ios resultados obtenidos se presentan en la tabla I.

En definitiva, los resultados expuestos permiten verificar que en la gran mayoría de los libros $(80 \%)$ no se hace explícito el problema general a resolver y que sólo en un tercio de los libros de universidad se encuentra alguna proposición que sirva para formular un problema estructurante.

Consecuentemente con lo anterior, la introducción de nuevos conceptos como el de campo y potencial eléctricos se hace en la mayoría de los textos de forma arbitraria (83\%) sin mencionar la problemática de la que surgieron, y a la que intentan dar solución. Esta situación es más grave cuando se trata de mencionar las diferentes formas de ver la interacción eléctrica según el modelo utilizado, sea coulombiano o de campo, y es coherente con la presentación del concepto de potencial eléctrico, donde sólo el $5 \%$ de los textos analizados menciona de forma explícita las ventajas del modelo «energetista» para el análisis de la causalidad del movimiento de las cargas. En el caso del concepto de campo eléctrico, solamente algo más de un tercio (35\%) de los libros de tex to lo diferencian explícitamente de la teoría newtoniana de acción a distancia.

En todos los libros de bachillerato analizados sólo sc menciona la teoría eléctrica actual, aunque, en algunos casos, se realizan algunas referencias a la historia de la electricidad. En cambio, en Ia mitad de los libros de

Tabla I

Insuficiencias y carencias ontológicas y epistemológicas presentes en la organización y secuenciación de contenidos de electrostática en los libros de texto.

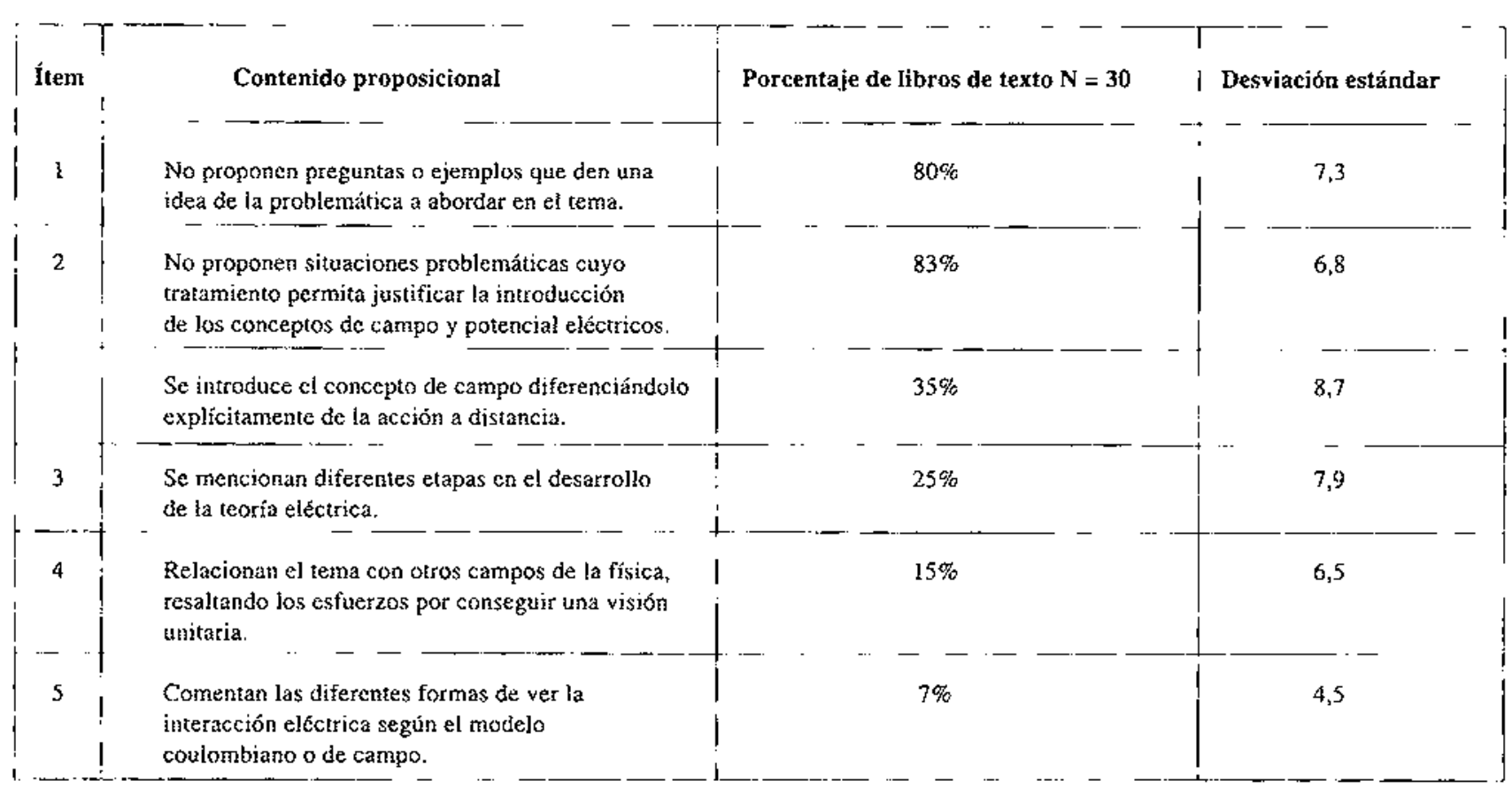


Tabla II

Carencias epistemológicas observadas en la organización y secuenciación de contenidos de electrostática propucstas por el profesorado.

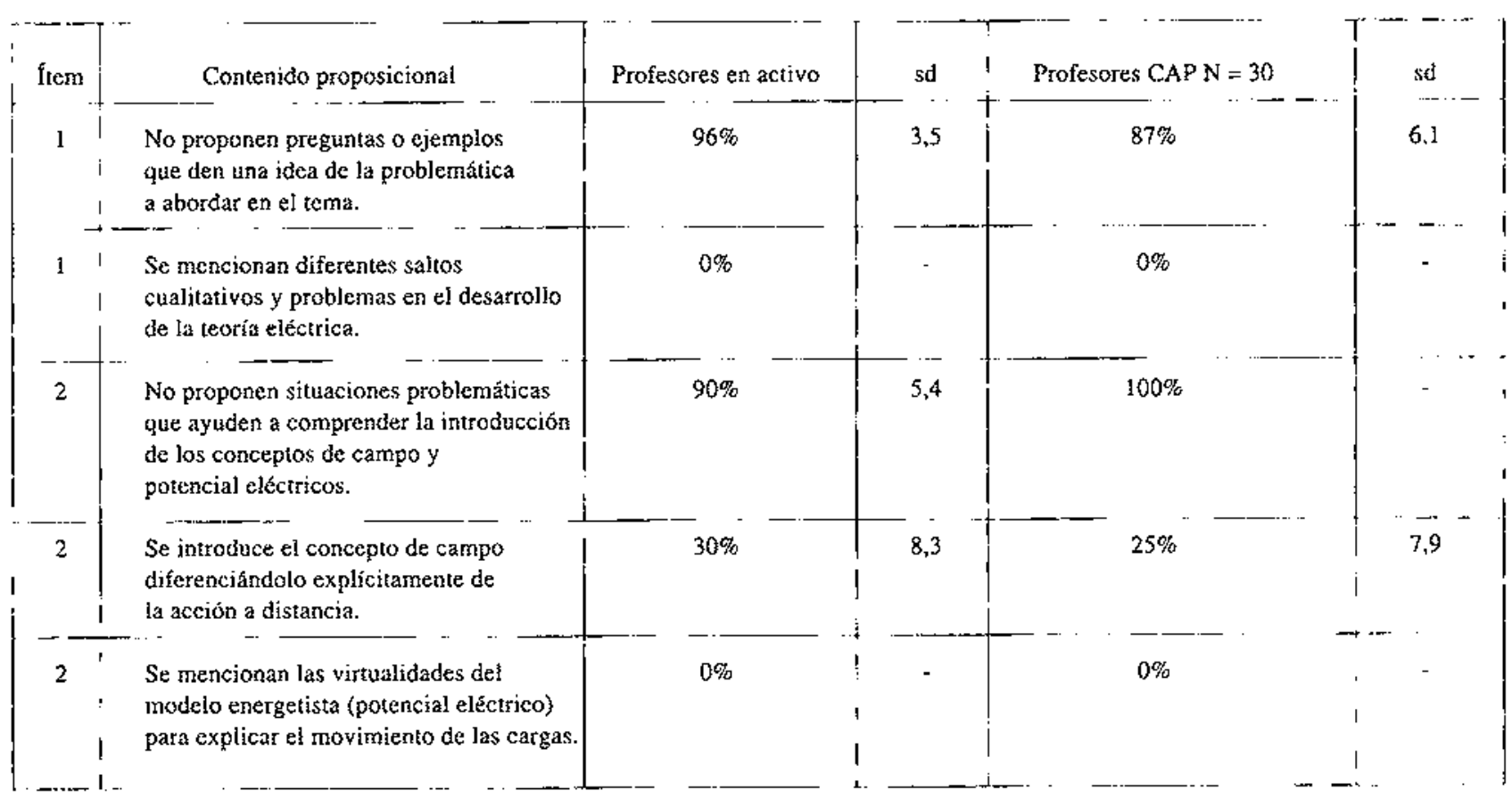

universidad se mencionan las diferentes etapas del desarrollo de la teoría eléctrica, pero esta referencia se hace de forma lineal y acumulativa, sin hacer mención de los problemas que surgieron. Los libros analizados están más interesados en explicar la lógica interna de la disciplina que en indicar las dificultades habidas y los logros obtenidos al superarlas. Respecto a los libros de física general analizados hay que resaltar que la mayoría son traducciones de libros representativos de los textos de física utilizados en el mundo anglosajón, y que estas carencias también están presentes en estos libros de lengua inglesa (Strube, 1988). Fn definitiva, podemos concluir que la presentación de los contenidos de electrostática se hace desde una visión acumulativa y acrítica de la misma.

Por último es necesario resaltar que la gran mayoría de Ios libros de tex to (95\%) no tienen en cuenta cl cambio ontológico que se produce, en la forma de concebir la interacción eléctrica de una visión coulombiana a otra de campo eléctrico.

\section{Resultados del análisis del cuestionario y de las entre- vistas realizadas al profesorado}

En el primer ítem del documento 2 se solicitaban comentarios sobre la secuencia de contenidos que llevaban o podrían llevar a cabo y, en el segundo f́tem, sobre la forma en que introducian los conceptos de campo y potencial eléctricos. Ios aspectos que el profesorado encuestado no tiene en cuenta en la organización y secuenciación de los contenidos se pueden clasificar en Ia tabla $\mathrm{H}$.

De acuerdo con la tabla se puede decir que las secuencias propuestas no indican cuáles son los problemas a abordar en el tema, presentando una serie de conocimientos de forma acumulativa acrítica (96\% de profesores en activo y $87 \%$ de profesores en formación). EI criterio seguido por el profesorado, tanto de bachillerato como de universidad, para explicar la secuenciacion realizada, es la lógica interna de la disciplina, que viene reflejada en las secuencias de los Iibros de texto. Fjemplos de este tipo de respuestas, los podemos ver en las siguientes dos transcripciones de las entrevistas realizadas a un profesor de universidad y a otro de bachillerato:

\section{Ejemplo}

$\mathrm{E}$ (Entrevistador): El orden de la secuenciación que has puesto, ¿a qué se debe? ¿Qué criterio has utilizado?

P (Profesor): El orden lógico. Primero, hablamos de la carga, luego de qué interacciones hay entre cargas eléctricas; después cómo sabemos que en una región del espacio hay influencia eléctrica; y al final de las implicaciones energéticas. No sé, me parece el orden lógico.

\section{Ejemplo}

E: ¿Cómo realizas la secuenciación del tema de clectrostíticá ¿Qué criterios utilizas?

P: Más o menos de lo más sencillo es decir; de los aspectos concretos o cualitativos, como atracciones y repulsiones o cuerpos cargados por frotamiento, a los aspectos más difíciles, como campo eléctrico o energía. Siguiendo el orden de la teoría. 


\section{E: ¿Puedes concretarlo un poco más?}

P: Sí, empiezo por lo más sencillo. Lo que más entienden ellos, en principio, es la fuerza porque hay dos cargas, y la ley de Coulomb. Luego, se introduce el campo que es mucho más difícil. Y después, el concepto de potencial.

En casi ninguna de Ias secuenciaciones, los profesores de bachillerato hacen referencia a los problemas que dan pie a introducir los nuevos conceptos de campo y potencial eléctrico, así como tampoco a las posibles ventajas de los mismos frente a los ya conocidos como el concepto de fuerza de la ley de Coulomb y las diferentes visiones ontológicas de cada modelo. Las entrevistas mantenidas con los profesores de bachillerato muestran cierto conocimiento de las definiciones operativas de campo y potencial eléctricos, pero desconocen la necesidad de su introducción e incluso dudan de su utitidad. Sin embargo, la mayoría de los profesores de universidad entrevistados indican explícitamente las limitaciones del modelo coulombiano y la necesidad de introducir el modelo de campo, observándose un conocimiento más profundo de la materia a enseñar, aunque ello no implica que resalten los saltos cualitativos y el cambio ontológico que supuso el paso de un modelo a otro en sus secuenciaciones. Esto queda reftejado en las siguientes transcripciones de las entrevistas correspondientes a un profesor de bachillerato (ejemplo 1) y a dos de universidad (ejemplos 2 y 3 ):

1. Ejemplo de respuesta que refleja la falta de conocimiento en profundidad del cuerpo teúrico de la disciplina y del cambio ontológico al pasar de un modelo coulombiano a otro maxwelliano

Entrevistador (E) ¿ Cómo justificas la introducción del concepto de campo?

Profesor (P): Presentar la idea de campo es muy, muy difícil, por eso de que el campo es como algo que no existe, que no se ve y que es el causante de que se manifiesten fuerzas entre objetos. Hombre, no es fácil que lo vean... en cierto modo acaban haciendo un acto de fe ¿no? Por mi parte lo presento como... yo que sé, a veces parece magia: se han inventado una cosa para justificar que cxisten fuerzas.

E: ¿,Pero tú les indicas a los alumnos las ventajas de introducir el campo frente, por ejemplo, a utilizar la ley de Coulomb? ¿Explicas las ventajas o inconvenientes de uno respecto al otro?

P: ¿Ventajas? Como te diría yo... yo no hablaría de ventajas, yo prefiero presentarlo como una forma de explicar que un objeto le haga una fuerza a otro aunque no haya nada que los una. En mecúnica han visto planos inclinados, poleas, cuerdas; entonces el hecho de que un cuerpo pueda ejercer una fuerza sobre otro que no está en contacto con él se explica muy fácil si está unido por una cuerda o algo material, ¿no? Pero en el espacio no hay nada material; o sea, en el vacío, un objeto es capaz de tirar de otro, entonces, bueno, pues aparece el concepto de campo. Así explicas la interacciớn y parece convincente.

En mi opinión, el concepto de campo es realmente problemático. ¿Por qué no quedarse con la ley de Coutomb y las fuerzas simultáneass que actúan a distancia? ¿Qué utjlidad tiene el concepto de campo eléctrico? Eso de la «modificación del espacio» por una carga no les dice nada a los alumnos.

2. Ejemplo de respuesta que refleja conocimiento del marco teórico actual de la disciplina, pero que no resalta los saltos cualitativos del desarrollo de la misma
Entrevistador (E): ¿Cómo justificas la introducción de los conceptos de campo y potencial eléctricos?

Profesor (P): Bueno, hay que tener cuidado al pasar de la ley de Coulomb, que es una interacción a distancia, al concepto de campo, y dejar claro las ventajas que supone este concepto. Entre estas ventajas tenemos la visión energética de la interacción, que nos permite definir la energía potencial eléctrica al ser la interacción de carácter conservativo.

E: ¿Reflejas estas diferencias en tu programación, haciendo apartados para las dificultades y para las ventajas?

$P$ : No, apartados no hago, sólo al empezar la lección hago una pequeña introducción general sobre el desarrollo de la teoría eléctrica.

3. Ejemplo de respuesta que refleja la falta de conocimiento del cambio ontológico entre el modelo cotilombiano y de campo eléctrico

Entrevistador (E): ¿Cómo introduces el concepto de campo eléctrico?

Profesor (P): Bueno, aquí hablamos de la interacción eléctrica asociada a una zona del espacio. Se empieza diciendo que la carga eléctrica modifica la zona del espacio próxima a ella y después se desarrolla la teoria y sus leyes.

E: ¿Explicas las ventajas de ver la situación desde un punto de campo eléctrico?

P: Bueno, decimos que es una forma más sencilia de operar y resolver problemas en determinadas ocasiones. Se les enseña a resolver problemas utilizando la ley de Gauss, aunque sólo es efectiva para simetrías muy concretas. Además, el teorema de Gauss es en definitiva la ley de Coulomb; es decir, es necesario que la interacción sea inversamente proporcional al cuadrado de la distancia.

Respecto del análisis de las respuestas obtenidas en el ítem 3 del cuestionario (documento 2), relativas a la contextualización teorica de los conceptos de campo y potencial eléctricos, hay que resaltar que sólo una minoría del profesorado de bachillerato $(10 \%)$ menciona la teoría max welliana para enmarcar dichos conceptos. La gran mayoría de respuestas se sitúan en un marco newtoniano ( $45 \%$ profesores de bachillerato y $65 \%$ profesores en formación) o en el modelo atómico de la materia de Bhor (30\% profesores de bachillerato y $10 \%$ profesores en formación). Más de un tercio de los profesores en formación no responđe a esta cuestión, indicando que al no enseñar el tema no se han planteado la pregunta.

Los resultados del análisis de las entrevistas a profesores de bachillerato son convergentes con los de las encuestas. Sin embargo, en las entrevistas realizadas a los profesores de universidad, la mayoría de ellos menciona el marco integrador más actual, indicando que se trata de un modelo de interacciones entre cuerpos cargados que se explica mediante la teoría cásica de MaxweIl. No obstante, esto no implica que destaquen las diversas reformulaciones habidas en el desarrollo de la teoría. Presentamos a continuación algunas trascripciones de las entrevistas correspondientes a dos profesores de bachiIlerato (ejemplos 1 y 2) y a uno de universidad (ejemplo 3 ), que ilustran algunas de las posturas expuestas. 
1. Ejemplo de respuesta que indica como marco teórico el modelo newtoniano

Entrevistador (E): Respecto del modelo teórico, ¿cuál es el que utilizas para enmarcar los conceptos del tema?

Profesora (P): El marco teórico en que sitúo la electrostática es la teoría de Coulomb, que es cl único que conozco, por cierto.

E: ¿No conoces ningún otro modelo teórico para las teorías eléctricas?

P: Bueno, luego estaría la teoría cuántica o la relativista, pero en la teoría clásica tenemos el modelo newtoniano.

\section{Ejemplo de respuesta que no sabe exactamente cómo indicar el modelo teúrico}

Entrevistador (E): Respecto del modelo teórico, ¿cuál es cl que utilizas para enmarcar los conceptos del tema?

Profesor (P): Bueno, no sé exactamente a lo que te reficres, pero creo que sería el modelo de Bhor para la estructura eléctrica de la materia.

F: Y luego, cuando empiezas a utilizar las fuerzas y defines la ley de Coulomb, y después para definir el campo y el potencial cléctricos, ¿ahí qué marco teórico utilizas?

P: ¿Para la ley de Coulomb? No sé. La ley de Coulomb, la introducriamos en ese modelo, se supone. Claro, lo que pasa es que esto yo no me to planteo; Io de Coulomb o el campo se ven como cosas aisladas, yo por lo menos.

\section{Ejemplo de respuesta que indica como marco teórico el} actualmente aceptado en la mecúnica clasica

Fntrevistador: ¿Qué modelo teórico utilizas para explicar los conceptos de electrostática?

Profesora: Sería un modelo de interacciones debido a la carga eléctrica que contiene las leyes de Maxwell; es decir, contempla la teoria de campo y de la energía.

E: ¿Indicas las diferencias entre este modelo max welliano y el modelo de interacción newtoniano?

P: Bueno, en realidad todo está dentro del marco téorico de la mecánica clásica. Las leyes de Maxwell resumen la interacción electromagnética dentro del marco clásico. luego, se marcan las diferencias entre este marco cásico, y el cuántico y el relativista.

Del análisis de las respuestas al cuestionario y de las entrevistas se puede concluir que, aunque los profesores de universidad poseen un mayor dominio de la estructura actual de la disciplina, ambos colectivos presentan insuficiencias en la construcción de la electrostática y del trabajo científico basadas en una concepción aproblemática y acumulativa lineal de lo conocimientos científicos, y en la no consideración del cambio ontológico que supuso pasar de un modelo a otro. Al mismo tiempo, podemos apreciar que los resultados obtenidos sobre las concepciones del profesorado se corresponden, dentro de los márgenes de fiabilidad estadística, con los obtenidos en el análisis de textos sobre carencias epistemológicas en la presentación de la organización y se- cuenciación de contenidos. Esto nos permite afirmar la verosimilitud de nuestra hipótesis en lo que se refiere a estas carencias en la enseñanza habifual de la clectrostáticáa.

\section{CONCLUSIONES Y PERSPECTIVAS}

Una de las características de la naturaleza de la ciencia es la consideración de las teorías y modelos como productos sociohistóricos sometidos a discusión y reelaboración por la comunidad científica (Kuhn, 1971). En contraposición, acabamos de ver que frecuentemente en la enseñanza habitual y en los libros de texto se presenta una visión acumulativa y acrítica que no tiene en cuenta la diferentes formulaciones y saltos cualitativos que se dieron en el desarrollo de la teoría como, por cjemplo, al pasar directamente de una visión newtoniana de la clectricidad (Coulomb) a una visión energetista (campo eléctrico) sin explicar la necesidad de este salto cualitativo (cambio ontológico).

Otra de las características de la naturaleza de la ciencia es el papel protagonista de los problemas como notor del desarrollo de las teorías, que permiten pasar a otras nuevas de mayor poder explicativo de los fenómenos considerados (Laudan, 1984). Frente a esta concepción de la ciencia que viene a converger con la anterior, hemos visto que en la enseñanza habitual y en los libros de texto se presenta una visión aproblemática que no explica, por ejemplo, por qué es necesario introducir el concepto de campo o el de potencial eléctrico.

Por último es necesario resaltar el cambio ontológico que se produce de una visión conlombiana a otra de campo eléctrico en la forma de concebir la interacción eléctrica, y que no suele tenerse en cuenta en la enseñanza habitual de estas teorías. La carencia de esta nueva visión ontológica lleva a considerar la teoría de canpo como una forma muy «abstracta» de justitificar las interaccioncs eléctricas y, en consecuencia, a no enfatizar la necesidad de su introducción en la propia enseñanza.

A la luz de estos resultados y dentro del matco constructivista de aprendizaje se plantea como desafío el planteamiento de estrategias que aborden la enseñanza-aprendizaje de cstos temas de fisica y, en concreto, de electrostática. En este reto se habrán de idear investigaciones donde se planteen situaciones problemáticas en un entorno de aprendizaje por investigación, donde se favorezca que los estudiantes conozcan nuevos conceptos cientificos de mayor poder explicativo, se familiaricen con las características fundamentales del trabajo científico y sus exigencias epistemológicas, así como con las distintas maneras de ver el mundo que se derivan de los diferentes modelos explicativos (Gil et al., 1991; Guisasola, 1996). 


\section{REFERENCIAS BIBLIOGRÁFICAS}

AMPËRE, A.M. (1827). Mémoire sur la théorie mathématique desphénomènes électro-magnetiques déduite del expérience. Reeditado por Blanchard en I958. París.

BENSHEGUIR, A. (1987). Formation des concepts d'electrocinetique: un point de vue historique. Actas del Congreso $S F P$ de Estrasburgo.

BERKSON, W. (1985). Las teorias de los campos de fuerza desde Furaday hasta Einstein. Madrid: Alianza Editorial.

CHI, M.T.H. (1991). Conceptual change within and across ontological categories: Examples from Jearning and discovery in science, en Giere, R. (ed.), Cognitive models of Science: Mimesota Studies in the philosophy of Science. Minnesota: University of Minnesota Press.

COUI.OMB, C. A. (1784-1806). Colection de Memoires relatifs a la Physique, publicado en 1884 por la Real Sociedad Francesa de Física, Vol I. París.

IJRIVER, R. (1989). Students' conceptions and the learning of science. International Journal of Science Education, 11(5), pp. $481-490$.

DUIT, R. (1993). Research on student's conceptions-developments and trends, documento presentado en Third Intemational Seminar on Misconceptions and Educational Strategies in Science and Mathematics. Cornell: thaca.

IJUSCHI., R.A. y GITOMER, D.H. (1991). Epistemological perspectives on conceptual change: implications for Educational Practice. Journal of Research in Science Teaching, 28(9), pp. 839-858.

EYLON, B.S y GANIEL, U. (1990). Macro-micro relationships: the missing link between electrostatics and electrodynamics in students" reasoning. International Journal of Science Education, 12(1), pp. 79-94

FARADAY, M. (1821). Historical sketch of electromagnetism. Annals of Philosophy, 18, pp. 195-200.

FARADAY, M. (1839, 1844, 1855). Experimental researches on electricity, 3 vol. Royal Socicty. Londres. Trad. cast. (1971). Investigaciones experimentales de electricidad, serie I a $V$, los fundamentales. Editorial Universitaria de Buenos Aires.

FRANKIIN B. (1988). Experimentos y observaciones sobre electricidad. Madrid: Alianza Universidad

FURIÓ, C. (1994). La enseñanza de las ciencias como investigación: un modeloemergente. Proceedings International Conference "Science and Mathematics Education for the 21 st. Century: Towards innovatory approaches». Concepción. Chile: Universidad de Concepción.

FURIÓ, C. y GUISASOLA, J. (1993). ¿Puede ayudar la historia de la ciencia a entender por qué los estudiantes no comprenden los conceptos decarga y potencial eléctricos? Revista Española de Fisica, 7(3), pp. 46-50.

GALIII, I. (1995). Mechanics background influences students' conceptions in electromagnetism. International Journal of Sciance Education, 17(3), pp. 371-387.

GIL, D. (1993). Contribución de la historia y filosofía de la ciencias al desarrollo de un modelo de enseñanza -aprendizaje como investigación. Enseñanza de las Ciencias, I1(2), pp. 197-212.
GIL, D., CARRASCOSA, J., FURIÓ, C. y MARTÍNEZTORREGROSA, J. (1991). La enseñanza de la ciencias en la educación secundaria. Barcelona: Horsori-ICE Universitat de Barcelona.

GUISASOLA, J. (1996). Análisis crítico de la enseñanza de la electrostática en el bachillerato y propuesta altemativa de orientación constructivista. Tesis doctoral. Universidad del País Vasco.

GUISASOLA, J. y FURIÓ, C. (1994). Dificultades en el aprendizaje significativo de algunos conceptos de electrostática. Investigación en la Escuela, 23, pp. 103-114.

HARMAN, P.M. (1990). Energía, fuerza y materia. El desarrollo conceptual de la física en el siglo $x I x$. Madrid: Alianza Editorial

HEILBRON, J.L. (1979). Electricity in the $17^{\text {th }}$ and $18^{\text {th }}$ centuries. California: University of California Press. Berkeley y LoS Angeles.

HOLTON, G. y ROLI.ER, D. (1963). Fundanentos de la fisicat moderna. Barcelona: Editorial Reverté.

IZQUIERDO, M. (1992). Reconsidering the sciences curriculum starting from contemporary (converging) models of science and cognition: a research program. Proceedings of the second International Conference on History and Philosophy of Science in Science Teaching, pp. 517-529. Kingston. Canadá.

JONG de, O. (1995). Classroom protocol analysis: A fruitfu! method of research in science education. Proceedings of the second Ph. D. summer school. Tesalónica: D. Psillos.

KUHN, T. (1990). La estructura de las revoluciones cientificas. México: Fondo de Cultura Económica.

LAUDAN, L. (1984). Science and values: the aims of science and their role in science debate. Berkeley: University of California Press.

MASON, S.F. (1985). Historia de las ciencias. La ciencia del siglo xwm, tomo 3. Madrid: Alianza Editorial.

MATTHEWS, M.R. (1990). History, philosophy and science teaching: a rapprochement studies. Science Education, 18, pp. 25-5I.

MAXWELL, J.C. (1865). Dynamic theory of the electromagnetic field. Philosophical transactions, 155, pp. 456-512. Reedicion en The scientific papers of James C. Maxwell. Cambridge (1980).

MORTIMER, E.F. (1995). Conceptual change or conceptual profile changc. Science \& Education, 4, pp. 267-285.

NARDl, R., y PESSOA de CARVHALO, A.M. (1990). A genese, a psicogenese e a aprendizagem do conceito de campo: subsídios para a construção do ensino desse conceito. Caderno Catarinense do Ensino de Física, 7, pp. 46-69.

NERSESSIAN, N.J. (1995). Should physicist preach what they practice? Science \& Education, 4, pp. 203-226.

PORI.AN, R. (1993. Constructivismo y escuela. Hacia un modelo de enseñanza-aprendizaje basado en la investigación. Sevilla: Díada.

POSNER, G., STRIKE, K., HEWSON, P. y GERTZOG, W (1982). Accomodation of a scientific concepcion: Toward a 
theory of conceptual change. Science Education, 66, pp. $211-227$.

RESNICK, L.B. (1983). Mathematics and Science Learning: a new conception. Science, 220 , pp. $477-487$.

RODRÍGUEZ, M. (1983). La electricidad en el siglo xvir. Curso de conferencias sobre historia de la fisica hasta el siglo $x I X$. Real Academia de las Ciencias Exactas, Físicas y Naturales.

SALTIEL, E. y VIENNOT, I. (1985). ¿Qué aprendemos de las semejanzas entre las ideas históricas y el razonamiento espontáneo de los estudiantes? Enseñanza de las Ciencias, 3(2), pp. 137-144

SOI.BES, J. y MARTÍ, J. (1991). Análisis de la introducción del concepto de campo. Revista Española de Física, 5(3).
STRUBE, P. (1988). The presentation of energy and fields in physics texts: a case of literary inertia. Physics Education, 23 , pp. 366-371.

TATON, R. (1988). Historia general de las ciencias. Ediciones Orbis.

THUILLIER, P. (1989). De la filosofía al electromagnetismo: el caso Oersted. Mundo Científico, Vol. 10, p. 102.

VIENNOT, L. y RAINSON, S. (1992). Students' reasoning about the superposition of clectric fields. International Journal of Science Education, 14(4), pp. 475-487.

WHITTAKER, E. (1987). A History of the Theories of Aether and Electricity, Vol. 1 . The classical theories. Thomas Publishers. American Institute of Physics. EEUU.

[Artículo recibido en mayo de 1994 y aceptado en noviembre de 1996.]

\begin{abstract}
ANEXO
DOCUMENTO 1. ESTADILLO PARA ANÁLISIS DE LIBROS DE TEXTO SOBRE LAS GRAVES CARENCIAS EPISTEMOLÓGICAS EN LA ORGANIZACION Y SECUENCIACIÓN DE LOS CONTENIDOS
\end{abstract}

1. ¿Hay en el tema introductorio de la electricidad (electrostătica) algún contenido explícito cn forma declarativa o interrogativa relativo a la problemática que se va a abordar (que puede dar una concepción preliminar de lo que se va a estudiar)?

Síl NO

2. ¿Se presentan situaciones problemáticas con objeto de hacer ver la necesidad de introducir los nuevos conceptos que se van a definir?

Sí/ NO

3. ¿Sc hacc algán comentario histórico sobre el desarrollo de los conceptos básicos de la electricidad (carga, campo y potencial eléctricos) considerando, en particular, algunas de las diferentes explicaciones que se dieron a lo largo de la historia de la electricidad hasta llegar al modelo teórico actual?

SÍ/ NO)

4. ¿Hay alguna conexión explícita de cualquier tipo entre la introducción del tema de electrostática y algún capítulo anterior?

Sí/ NO

5. ¿Se hace algún comentario sobre las diferentes formas de ver la interacción cléctrica en el modelo coulombiano y en el de campo eléctrico? 
DOCUMENTO 2, CUESTIONARIO PARA DETECTAR VISIONES DEFORMADAS DE LA CIENCIA EN LA ORGANIZACION Y SECUENCIACIÓN DE LOS CONTENIDOS DE ELECTROSTÁTICA QUE REALIZAN LOS PROFESORES

En relación con la próxima puesta en marcha del nuevo bachillerato y con la intención de mejorar la enseñanza, estamos recogiendo la opinión del profesorado sobre aquellas partes de la física que tienen más dificultades de aprendizaje. En particular te agradeceríamos que contestaras el siguiente cuestionario relacionado con el bloque de electrostáticá.

1. Comenta la secuencia de contenidos que realizas o realizarías para el bloque de electrostática (para $3^{\circ}$ de BUP), entendiendo por tal los contenidos que van desde unas primeras ideas sobre electricidad hasta la introducción del concepto de campo y potencial eléctricos incluidos. Es importante que justifiques la secuenciación que eliges.

2. ¿Cómo justificas la introdución de nuevos conceptos como campo y potencial eléctricos?

3. Indica cuáles son los marcos teóricos que has utilizado para desarrollar los contenidos del bloque.

DOCUMENTO 3. ENTREVISTA GRABADA EN CASETE PARA CONTRASTAR QUE LA ORGANIZACIÓN Y SECUENCIACION DE CONTENIDOS REALIZADA POR LOS PROFESORES PRESENTA VISIONES ERRÓNEAS DE IAA CIENCIA

1. El profesor entrevistado comenta la secuenciación de contenidos que realiza en el bloque de electrostática, de acuerdo con la respuesta que ha escrito en el cuestionario (documento 2).

El entrevistador precisa algunos aspectos con las siguientes preguntas:

1.1. ¿Haces alguna introducción para explicar los problemas que se van a tratar en el tema?

I.2. ¿Qué criterio o criterios utilizas para secuenciar el tema de electrostática?

1.3. ¿Relacionas el bloque de electrostática con algún capítulo anterior?

1.4. ¿Consideras que Ios contenidos secuenciados se desarrollan de forma lineal de más «fácil» a más «difícil» o, por el contrario, existen pasos intermedios que exigen una explicación más detenida?

2. El profesor entrevistado comenta como justifica Ia necesidad de introducir los conceptos de campo y potencial eléctricos.

3. El profesor entrevistado explica el marco teórico donde sitúa la electrostática y los prerrequisitos de los alumnos.

El entrevistador precisa algunos aspectos con las siguientes preguntas:

3.1. ¿Podrías relatar algunos de los diferentes saltos cualitativos teóricos que se han dado en el desarrolio de la historia de la electricidad? 
